\title{
Identify of Granulicatella adiacens from blood cultures of a patient bearer of prosthetic valve
}

\author{
Raffaele Gargiulo', Carla Sabia', Stefano Giordani², Virginia Agnoletto ${ }^{3}$,Agostino Barozzi', \\ Fregni Sandra', Anna Mucci', Maria Olga Zirilli', Mario Sarti' \\ I Laboratorio Provinciale di Microbiologia Clinica, NOCSAE Baggiovara \\ 2 Consulente Infettivologo, NOCSAE Baggiovara \\ 3 UO di Cardiologia, NOCSAE Baggiovara \\ 4 Dipartimento di Scienze Biomediche, Università di Modena e Reggio Emilia
}

Key words: Granulicatella adiacens, prosthetic valve, identification

\begin{abstract}
Isolamento colturale di Granulicatella adiacens da emocolture in paziente portatore di protesi valvolare
\end{abstract}

\section{SUMMARY}

The clinical case studied concerns a woman 81 years old, with a history of prosthetic valve and mitral insufficiency, admitted to internal medicine ward of NOCSAE hospital as a result of a recurrent fever.

Due to the suspicion of endocarditis and with the aim to identify the presence of aerobic/anaerobic microorganisms, two set of blood cultures collected within 24 hours were sent to the Laboratory of microbiology. All the bottles were incubated into the Bact-Alert 3D System (bioMérieux). After an 19 hours incubation time, the samples were identified as positive by the automated system; consequently they cultured on a blood agar and selective media, according to our laboratory operational protocol.

In the same time Gram stain of the cultural broth revealed the presence of Gram positive cocci arranged in chains different in length. Since there wasn't an evident microbial growth on solid media after 24-48 hours of incubation, a new culture was carried out on blood and chocolate agar after the addition of Staphylococcus aureus ATCC 25923. After 24 hours of incubation it was possible appreciate the growth of tiny colonies around the $S$. aureus ones. These colonies were identified by Vitek2 and Api Rapid 32 Strep (bioMérieux) as Granulicatella adiacens. The results were confirmed by PCR and sequencing of the groESL gene. MIC values obtained by the means of E-test (bioMérieux) were: $0.016 \mathrm{mg} / \mathrm{L}$ for penicillin, $0.125 \mathrm{mg} / \mathrm{L}$ for cefotaxime, $1 \mathrm{mg} / \mathrm{L}$ for both vancomicin and levofloxacin. Resistance was observed for cloramphenicol (MIC=16mg/L). The timely communication of these findings, supported by clinical data like the appearance of vegetation on mitral valve highlighted by trans-oesophageal echocardiography, allowed to establish an adequate antibiotic therapy, rapid resolution of fever and normalisation of inflammatory parameters.

\section{INTRODUZIONE}

Granulicatella adiacens è un batterio del gruppo dei NVS (nutritionally variant streptococci) e può essere responsabile di infezioni gravi quali infezioni protesiche ed endocarditi (3). L'interazione del microrganismo con la fibronectina pare giocare un ruolo importante nell'instaurarsi dell'endocardite infettiva (4). Un precoce intervento chirurgico appare opportuno in pazienti con insufficienza cardiaca causata da distruzione valvolare.

Le endocarditi da NVS non sono molto frequenti, sono tuttavia associate ad elevati tassi di mortalità, complicanze ed insuccesso terapeutico. Tale quadro è in parte conseguenza della difficile identificazione microbiologica di questi microrgani$\operatorname{smi}(1,2)$.

Il caso clinico pervenuto alla nostra osservazione riguarda una donna di 81 anni, portatrice di protesi valvolare biologica ed affetta da insufficienza mitralica, ricoverata in un reparto internistico a causa di febbre recidivante. L'ecografia trans-esofagea mostrava la presenza di vegetazioni sui lembi mitralici della paziente. Sono stati raccolti, da accessi venosi distinti e in rapida successione, due set di emocolture per la ricerca di microrganismi aerobi ed anaerobi.

\section{MATERIALI E METODI}

I set di emocolture erano entrambi composti dal flacone per ricerca di aerobi associato al flacone per la ricerca di anaerobi (Bact/Alert FA e Bact/Alert FN, bioMérieux). I sistemi utilizzati per l'identificazione sono Vitek2 e Api Rapid 32 Strep. (bioMérieux).

\section{Corresponding author: Raffaele Gargiulo}

Laboratorio Provinciale di Microbiologia Clinica, Dip. di Patologia Clinica NOCSAE

Via Giardini, I 355 - 4I I 22 Baggiovara - Tel.: 0593962508 - Fax: 0593961249

E-mail: r.gargiulo@ausl.mo.it 
Mediante E-test (bioMérieux) utilizzato su terreno MHF (bioMérieux) sono state valutate le MIC nei confronti di: penicillina, cefotaxime, vancomicina, levofloxacina e cloramfenicolo.

Al fine di confermare l'identificazione del microrganismo isolato, il sequenziamento genico del gene groESL è stato condotto utilizzando lo strumento ABI Prism 3130 Genetic Analyzer (Applied Biosystems), secondo il metodo di Sanger, previa estrazione degli acidi nucleici con il metodo NucleoSpin Tissue kit (Machery Nagel), ed ottenimento dell' amplificazione dei relativi ampliconi (1). La sequenza ottenuta è stata allineata utilizzando il database blast/GenBank (http://www.ncbi.nlm.nih.gov/blast/Blast.cgi).

\section{RISULTATI}

La positivizzazione dei flaconi per emocoltura è avvenuta fra la prima e la terza giornata di incubazione. All'esame microscopico diretto con colorazione di Gram si sono osservati cocchi Gram positivi con disposizione a catenelle di lunghezza variabile (Figura I).

È stata quindi effettuata una semina della brodocoltura su Agar sangue ed Agar cioccolato. A fronte del mancato sviluppo di crescita microbica sui terreni solidi anche successivamente alle 48 ore di incubazione, si è proceduto ad una nuova semina del brodo colturale sui medesimi terreni, ma con l'aggiunta di uno striscio superficiale di una sospensione di S. aureus ATCC 25923.

Dopo 24 ore di incubazione è stato possibile osservare lo sviluppo di minuscole colonie, sempre localizzate in prossimità del ceppo di $S$. aureus ATCC 25923 (Figura II).

La colorazione di Gram, effettuata su tali microcolonie, ha confermato la presenza di cocchi Gram positivi con disposizione a catenella (Figura III).

L'identificazione biochimica, ottenuta prima con ViteK2 e successivamente confermata con Api32 Strep (bioMérieux), ha segnalato, con un valore di probabilità pari al $99 \%$, il profilo della specie Granulicatella adiacens.

Tale identificazione biochimica è stata confermata dall'analisi molecolare: il DNA batterico è stato sottoposto sia ad amplificazione genica per la ricerca del gene groESL (prodotto di amplificazione di dimensioni pari a $668 \mathrm{pb}$ ) che a sequenziamento.

L'E-test (bioMérieux) ha evidenziato bassi valori di MIC per: penicillina $(0.016 \mathrm{mg} / \mathrm{L})$, cefotaxime $(0.125 \mathrm{mg} / \mathrm{L})$, vancomicina $(1 \mathrm{mg} / \mathrm{L})$ e levofloxacina $(1 \mathrm{mg} / \mathrm{L})$, viceversa un valore elevato di MIC è stato riscontrato per il cloramfenicolo (16 $\mathrm{mg} / \mathrm{L}$ ).

Questi risultati hanno consentito di instaurare un'adeguata terapia antibiotica con rapida risoluzione della febbre e normalizzazione dei parametri flogistici.

\section{CONCLUSIONI}

Il caso riportato evidenzia come la diagnosi microbiologica di endocardite da Granulicatella adiancens comporti per il microbiologo la necessità di modificare $\mathrm{i}$ consueti protocolli operativi al

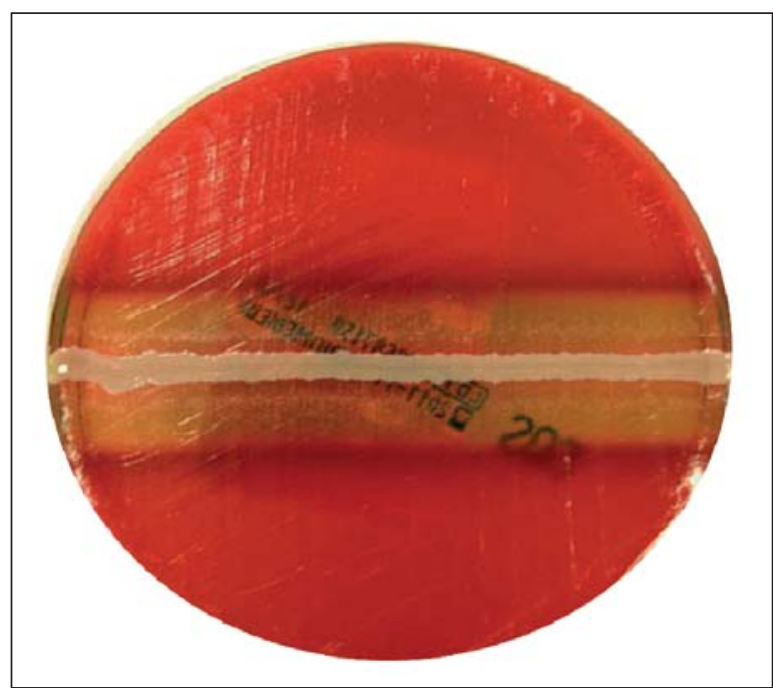

Figura II. Fenomeno del satellitismo.

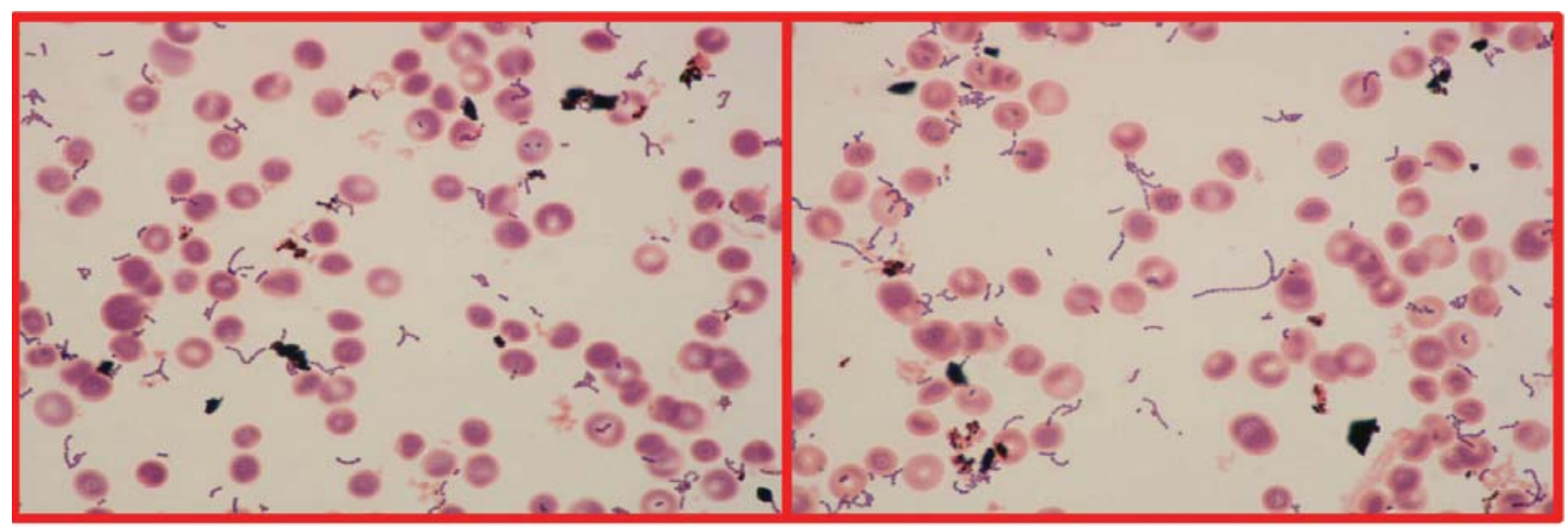

Figura I. Colorazione di Gram da brodo colturale. 


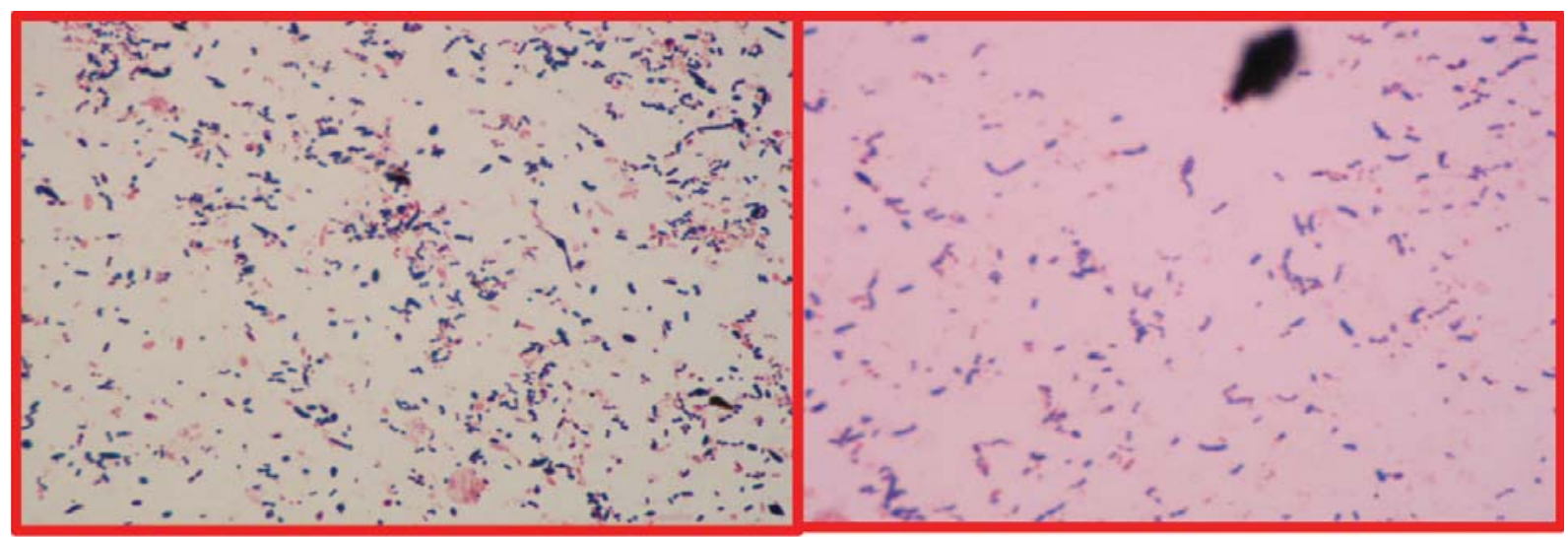

Figura III. Colorazione di Gram dalle colonie satelliti.

fine di ottenere l'isolamento colturale del microrganismo. Un'accurata diagnosi eziologica di patologie con decorso clinico subdolo quali le endocarditi batteriche appare di notevole impatto clinico e terapeutico.

\section{BIBLIOGRAFIA}

1. Casalta JP, Habib G, La Scola B, Drancourt M, Caus T, Raoult D. Molecular Diagnosis of Granulicatella elegans on the Cardiac Valve of a Patient with
Culture-Negative Endocarditis. J Clin Microbiol. 2010; 40 (5): 1845-7.

2. Hung WC, Tseng SP, Chen HJ, et al. Use of groESL as a target for identification of Abiotrophia, Granulicatella, and Gemella species. $J$ Clin Microbiol. 2010; 48 (10): 3532-8.

3. Lin $\mathrm{CH}$, Hsu RB. Infective endocarditis caused by nutritionally variant streptococci. Am J Med Sci. 2007; 334(4): 235-9.

4. Yamaguchi T, Soutome S, Oho T. Identification and characterization of a fibronectin-binding protein from Granulicatella adiacens. Mol Oral Microbiol. 2011; 26 (6): 353-64. 